\section{Adjustable Low Dynamic Pumps Based on Hydrogels}

Andreas Richter*, Christian Klenke, Karl-Friedrich Arndt

Institute for Physical Chemistry and Electrochemistry,

Dresden University of Technology, D-01062 Dresden, Germany

Email: Andreas:Richter@chemie.tu-dresdende

Summary: This paper discusses the suitability of hydrogel actuators as drives of automatic pumps for long-term drug release. We demonstrate that such actuators can automatic pumps for long-term drug release. We demonstrate that such actuators can
execute a defined task if several functional units are connected serially. Investigated execute a defined task if several functional units are connected serially. Investigated
functions of this pump are the adjustability of the time-delay from the initial operation up to the beginning of the drug release, opening the sterile drug ampoule, and the drug release at a specified timeframe. The described parameters to influence the pump behaviour are satisfying only for realization of continuous working devices. An outlook of the development of programmable pulsate pumps is given.

Keywords: medical pump, hydrogel actuator, drug release, fluidic component

\section{Introduction}

Smart hydrogels are able to change their volume by more than one magnitude in response to a lot of various sensitivities such as temperature, $\mathrm{pH}$ value, light, ion, and substance concentrations. Therefore, an enormous importance for many technological and scientific applications was expected [1]. Currently, some technological breakthroughs particularly in fluidics and sensorics could be indicated. Automatically [2-6] and electronically controllable [7] valves and microvalves have been described. Furthermore, a micro machined $\mathrm{pH}$ sensor based on smart hydrogels with a short insight of the sensor behaviour has been presented [8]

On the other hand, hydrogels without a smart behaviour also are important for many hightechnology applications. Particularly the principle of osmotic pumps [9], introduced in 1975, allowed the development of a new generation of drug delivery systems. For example, modern therapies of gastrointestinal tract diseases are hardly conceivably without such systems.

Automatic long-term working drives could be an interesting option to substitute some electromechanical drive systems, which needs auxiliary energy and includes a complicate mechanical design.

In present paper we investigated the suitability of long-time working osmotic hydrogel actuators as drives of drug release pumps. Such pumps could substitute the complex electromechanical medical pumps, which are commonly used.

\section{Experimental}

Materials

Sodium polyacrylate (PAAc-Na, HySorb C 7015 and M 3100) was obtained from BASF. Poly( $N$-isopropylacrylamide) (PNIPAAm) samples were prepared by following procedure. The crosslinking agent was $N, N^{\prime}$-methylenebisacrylamide (BIS). The initiator and accelerator for the polymerization reaction were potassium peroxidisulfate (KPS) and $N, N, N^{\prime}, N^{\prime}$-tetramethylethylenediamine (TEMED) respectively (both from Aldrich Chemical Co.). $N$-isopropylacrylamide (NIPAAm) and various amounts of BIS ( $1 \mathrm{~mol}-\%$ to $10 \mathrm{~mol}-\%$, for example BIS4 prepared with 4 mol-\%) were dissolved in deionized water. The total monome concentration was $0.53 \mathrm{~mol} / \mathrm{l}$. To initiate the polymerization reaction $0.3 \mathrm{~mol}-\%$ of KPS and TEMED were added to the oxygen free (bubbled with Nitrogen) solution. After polymerization (ca. $12 \mathrm{~h}$ at room temperature) the PNIPAAm gel was immersed in deionized water for about one week to wash out non-reacted reagents.

After drying the hydrogels particles with irregular shape were obtained by milling and subsequent fractionating into different particle sizes using test sieves.

\section{Pump Design}

Functional Requirements

Functional requirements of a pump for drug release are:

- a position independent operation;

- an adjustable time-delay from the initial operation up to the beginning the drug release;

- opening of the sterile drug ampoule immediately before starting the drug release;

- a drug release at a specified timeframe and at a defined volume.

To realize these features an automatic execution of a task sequence is necessary (see Fig. 1) 


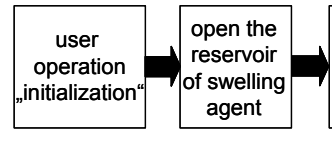

Figure 1. Automatic executable task sequence of a pump for drug release.

Pump Design and Functionality

The pump includes a trigger which allows their initialization (see Fig. 2). This switch can open the supply unit or swelling agent reservoir, respectively. A spring generates a pressure on the swelling agent reservoir. The resulting permanent hydrostatic excess pressure inside the swelling agent chamber enables a position independent loading of the actuator chamber or hydrogel actuator, respectively, with the swelling agent. The actuator chamber is encased by a highflexible foil.

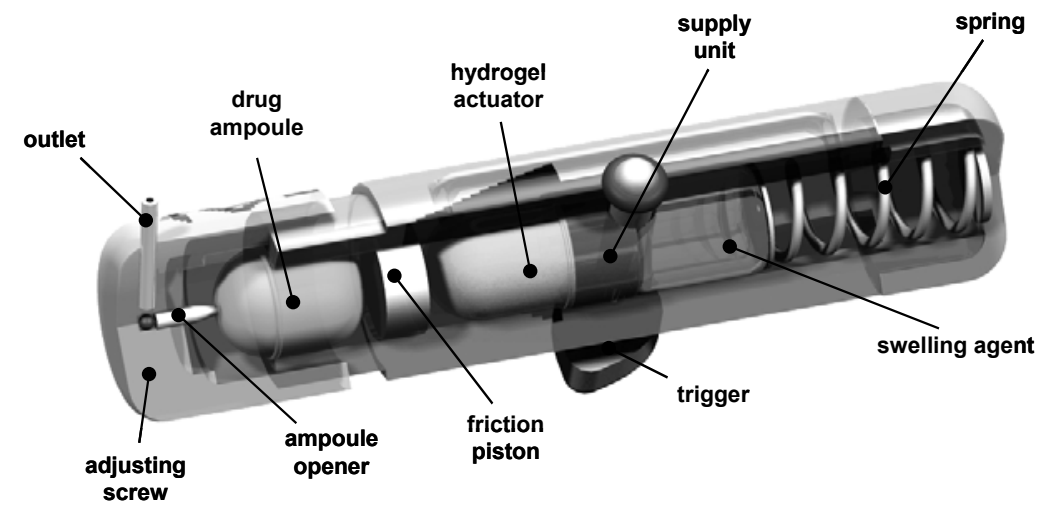

Figure 2. Design of a pump for drug release.

If the pump is initiated, the hydrogel actuator swells and stretches this foil. At the beginning of the swelling process the actuator must overcome a non-load distance or displaces a friction piston. The range of this distance is limited by the drug ampoule. The length of this way depends on the time behaviour of hydrogel actuator at the swelling process. Because the non-load distance can be adjusted using the adjusting screw, the time between pump initialization and starting the drug release is delayed. After passing the time-delay unit the actuator presses the drug ampoule at the opener to open the sterile ampoule. In the last step the hydrogel actuator crushes the ampoule content and the pump releases the drug.

\section{Results and Discussion}

Basic Characteristic of Hydrogel Actuator

The time behaviour of a hydrogel actuator depends on their smallest effective dimension. If the actuator material is based on particles, this dimension is the particle diameter. Furthermore, it will be influenced by the composition of hydrogel particles, their homogeneity, and the crosslinking density of hydrogel (see Fig. 3).

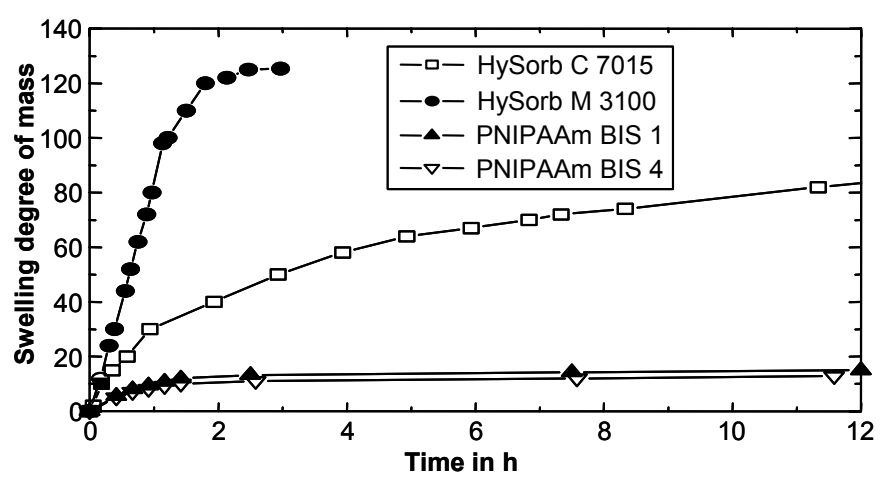

Figure 3. Swelling behaviour of various actuators based on hydrogel particles (diameter $\approx 500 \mu \mathrm{m}$ )

The basic time behaviour of such actuators follows an exponential function. The volume changes at the beginning of the swelling process are very fast. Approaching the swelling equilibrium the swelling rate decreases significantly. This feature is causing some problems for the pump design because a linear characteristic would be better usable.

Time-Delay

Firstly, the swelling characteristic must be adjusted for realization the time-delay function. A fast 
swelling process at the beginning results in a very long non-load distance, which is unacceptable because the length of the pump is increasing strongly. Using a hydrogel with a slow swelling behaviour such as C 7015 (see Fig. 3) a $18 \mathrm{~mm}$ long non-load distance is required to realize a time-delay of $2 \mathrm{~h}$ (see Fig. 4b). However, an applied opposing force to the swelling force could provide a decrease of the time - distance function. Their influence of the swelling behaviour of a hydrogel actuator is shown in Figure 4a.
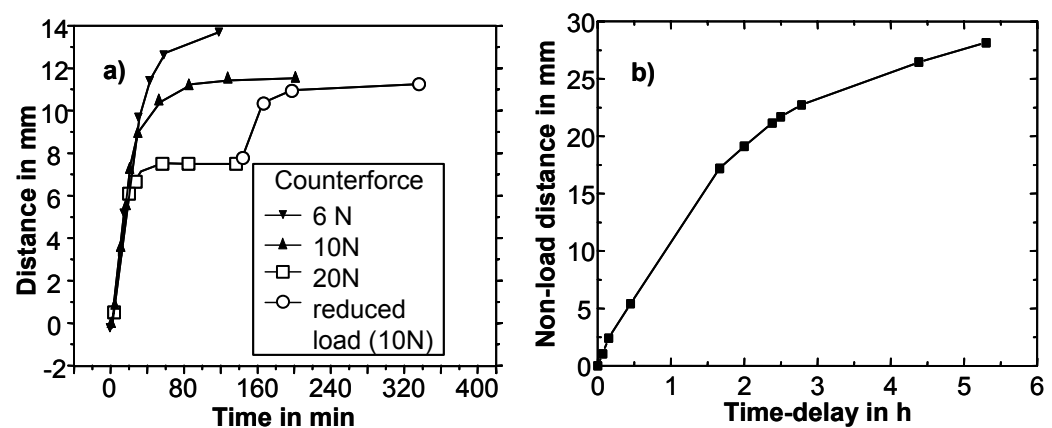

Figure 4. Dependency of time-delay from the distance: a) Time-delay distance depends on a applied opposing force; $b$ ) Time-delay as a function of a non-load distance.

The time-delay of $2 \mathrm{~h}$ can be realized with a short time-delay distance of about $8 \mathrm{~mm}$ using an opposing force of $20 \mathrm{~N}$. Furthermore, it can be seen that the hydrogel actuator obtains a swelling equilibrium which depends on the value of counterforce. The swelling process will restart if the opposing force is reduced. Hence, we utilize a friction piston which generates an opposing force to the swelling force. A nullifying of the counterforce is possible by increasing the inner diameter of pump body (strong decrease of the frictional force).

\section{Release Performance}

As it has been shown in Fig. 3, that the time and swelling behaviour of a hydrogel actuator is influenced by the composition of hydrogel particles, their homogeneity, the crosslinking density, and the particle size. Furthermore, the maximal volume change of the actuator depends strongly on their volume.
For a long-term release a characteristic nearly linear and a long-term swelling process of the hydrogel actuator is important. Both features can be realized through restriction of the supplying quantity of swelling agent (see Fig. 5). If the provided quantity of swelling agent is less than the hydrogel actuator needs for swelling to equilibrium, a linearization of the swelling characteristic as well as the release characteristic of the pump (see Fig. 5a) is resulted.
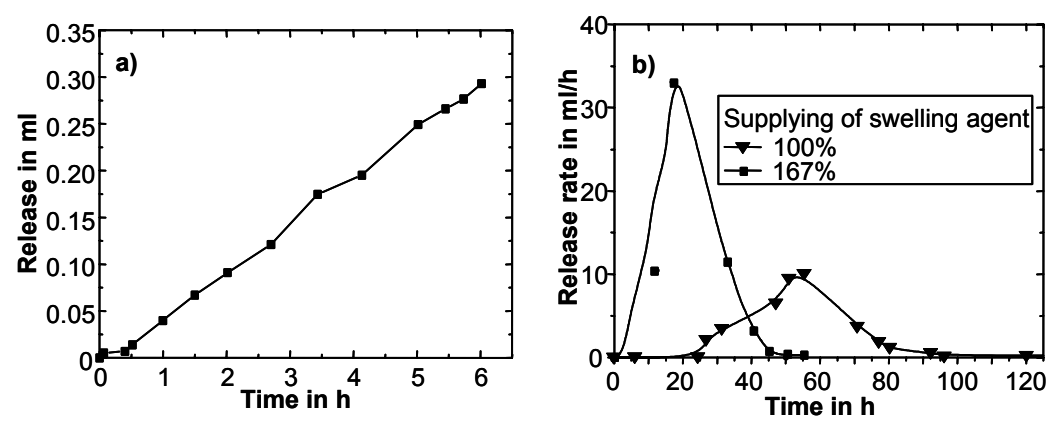

Figure 5. Release of a hydrogel driven pump depending on the supplied quantity of swelling agent: a) Linearization of the pump release; b) Release rate of pump depending on the supplying quantity of swelling agent.

On the other hand, an excess of the swelling agent decreases the maximal release of the pump and increases its release period (see Fig. 5 b).

\section{Operational Behaviour}

The behaviour of the drug release pump is shown in Figure 6. The time-delay function could successfully be realized. However, the use of a frictional force as a counterforce is problematic because the quality of the transition fit and the fabrication tolerance, respectively, must be very accurate. The ampoule opener process was also practicable. The membrane of the ampoule, which must be opened, should be relatively thin, otherwise the required opening force is all-too large. The pump can release a drug volume of about $500 \mu \mathrm{l}$ in $2 \mathrm{~h}$. 


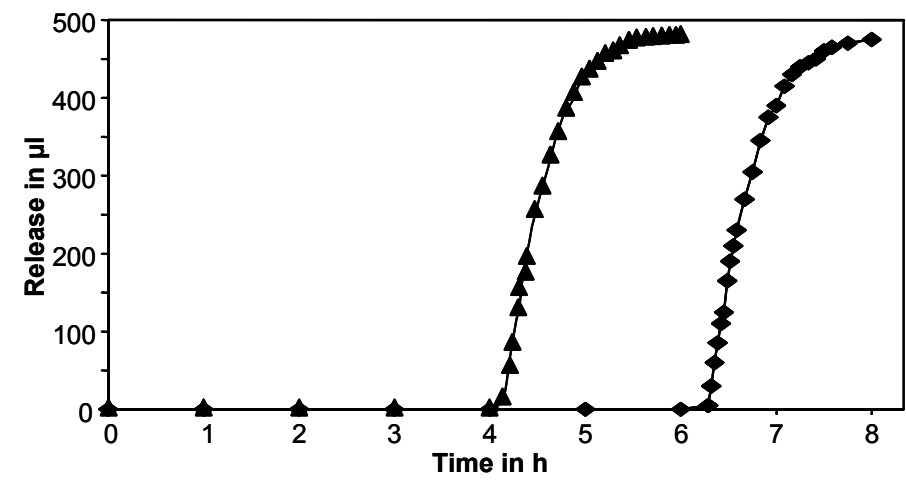

Figure 6. Operational behaviour of a drug release pump with adjustable time-delay.

\section{Conclusion and Outlook}

It was shown that hydrogel actuators can execute a defined task sequence if several functional units connected serially. Proved functions of a pump are the adjustable time-delay from the initial operation up to the beginning the drug release, opening of the sterile drug ampoule, and the drug release in a specified timeframe. The described parameters to influence the pump behaviour are satisfying only for realization of continuously working devices. Currently, such pumps will be developed ready to go into production.

To obtain a pulsating operational behaviour further requirements must be complied. Pulsations of drug release could be realized through serial connection of actuator materials with different swelling properties or by affecting the swelling agent supply. Interrupts are obtainable using water soluble swelling agent barriers. Further works will investigate the technical feasibility of programmable pulsatile working pump (see Fig. 7).

Such pump should include a programming unit which consists of some dials. These dials are showing several chambers which could be filled with various actuator materials, swelling agent barriers, etc. Through rotating the dials against each other task sequences are programmable.

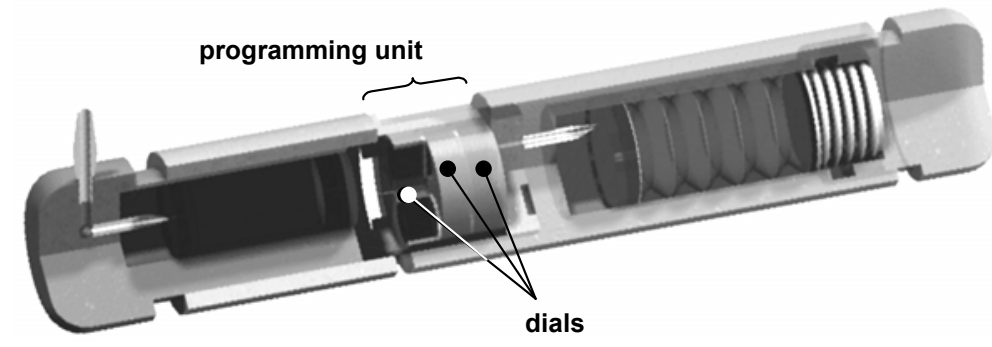

Figure 7. Programmable hydrogel driven pumps including a programming unit based on three dials.

\section{Acknowledgment}

Financial support has been provided by the Deutsche Forschungsgemeinschaft (SFB 287 "Reactive Polymers"). The authors would like to thank D. Kuckling and K. Kretschmer for the synthesis of PNIPAAm.

\section{References}

[1] A.E. English, E.R. Edelman, T. Tanaka, in: T. Tanaka (ed.): Experimental methods in polymer science: modern methods in polymer research \& technology. Academic Press, New York (2000), 547.

[2] K.-F. Arndt, D. Kuckling, A. Richter, Polym. Adv. Technol. 11 (2000) 8-12, 496.

[3] D. Kuckling, A. Richter, K.-F. Arndt, Macromol. Mater. Eng. 288 (2003) 2, 144

4] D.J. Beebe, J.S. Moore, J.M. Bacr, Q. Yu, R. H. Liu, C. Devadoss, B.-H. Jo, Nature 404 (2000), 588.

[5] A. Baldi, Y. Gu, P. E. Loftness, R. A. Siegel, and B. Ziaie, Proc. 15th. Int. IEEE Conference on M. A. B. Bas

(2003), 802

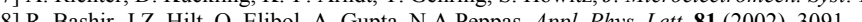

[9] F. Theeuwes: Elementary Osmotic Pump. J. Pharm. Sci. 64 (1975), 1987. 